\title{
Spin-orbital structure of the nucleon magnetic moment
}

\author{
Xiang-Song Chen, ${ }^{1,2}$ Di Qing, ${ }^{3,4}$ Wei-Min Sun, ${ }^{2}$ Hong-Shi Zong, ${ }^{5,2}$ and Fan Wang ${ }^{2}$ \\ ${ }^{1}$ Department of Physics, Sichuan University, Chengdu 610064, China \\ ${ }^{2}$ Department of Physics and Center for Theoretical Physics, Nanjing University, Nanjing 210093, China \\ ${ }^{3}$ Institute of Physics, Academy Sinica, Taiwan \\ ${ }^{4}$ CERN, CH-1211 Geneva 23, Switzerland \\ ${ }^{5}$ CCAST (World Laboratory), P.O. Box 8730, Beijing 100080, China
}

(Received 14 March 2003; revised manuscript received 22 December 2003; published 5 April 2004)

\begin{abstract}
How the nucleon magnetic moment originates from the quark spin and orbital motions is an important issue of the nucleon structure. The Gordon decomposition separates the quark vector current and magnetic moment into a spin and an orbital parts. We show that the spin part is related to the quark tensor charge, thus can be determined experimentally and computed reliably by lattice QCD. Knowledge of the spin-orbital structure of the nucleon magnetic moment would also shed light on the nucleon spin problem. As an example, we show that the spin part of the nucleon strangeness magnetic moment $\mu_{s}$ is negative. If the experimental result of a positive $\mu_{s}$ is confirmed, then the orbital motion of strange quarks must be important in the nucleon.
\end{abstract}

DOI: 10.1103/PhysRevC.69.045201

PACS number(s): 13.40.Em, 14.20.Dh

Structure of the nucleon magnetic moment $\mu_{N}$ is as important as, and closely related to, the structure of the nucleon spin. Historically, the naive quark model attributed all the nucleon spin to the quark spin and gave a quite successful explanation of $\mu_{N}$ in terms of the spin magnetic moment $\vec{\mu}_{\sigma}=(1 / 2 m) \vec{\sigma}$ of three constituent quarks. Today, however, we know that the quark spin only accounts for a small part of the nucleon spin, and it is under hot debate whether the rest is coming from the quark orbital motion or from the gluons [1]. Accordingly, the origin of $\mu_{N}$ must be reexamined.

Besides the physical significance in its own, knowledge of the spin-orbital structure of $\mu_{N}$ would also shed light on the nucleon spin problem. The $\mu_{N}$ structure is relatively simpler because only quarks contribute, while the angular momentum of gluons does not generate magnetism. Thus, if it were revealed that the quark spin magnetism cannot fully account for the nucleon magnetism, then the quark orbital motion must be important. Sadly, the relatively simpler spin-orbital structure of $\mu_{N}$ did not receive much attention in the past, and it was (to the best of our knowledge) never discussed how to determine it experimentally. In the literature, there have been arguments that the quark magnetic moment is correlated not with the quark helicity distribution, but more reasonably with the quark tensor charge $[2,3]$. This paper will present a first-principles analysis of the spin-orbital structure of $\mu_{N}$ and discuss the physical implications.

The spin-orbital separation of $\mu_{N}$ is obtained by the Gordon decomposition [4], which separates the quark vector current $j^{\mu}=\bar{\psi} \gamma^{\mu} \psi$ into a convection part and a spin part:

$$
\bar{\psi} \gamma^{\mu} \psi=\frac{i}{2 m} \bar{\psi} \stackrel{\leftrightarrow}{D^{\mu}} \psi+\frac{1}{2 m} \partial_{\nu}\left(\bar{\psi} \sigma^{\mu \nu} \psi\right) \equiv j_{C}^{\mu}+j_{S}^{\mu},
$$

where $m$ is the mass of the quark field and $\stackrel{\leftrightarrow}{D^{\mu}}=D^{\mu}-\stackrel{\leftarrow}{D^{\mu}}$ is the covariant derivative. The Gordon decomposition follows directly from the equation of motion. In case of free field, $D^{\mu}$ is replaced with $\partial^{\mu}$.
Applying Eq. (1), the magnetic moment operator $\vec{\mu}$ $=\frac{1}{2} \int d^{3} x \vec{r} \times \vec{j}$ can be separated into an orbital part and a spin part:

$$
\vec{\mu}=\frac{1}{2 m} \int d^{3} x \vec{r} \times \bar{\psi} \frac{1}{2 i} \stackrel{\leftrightarrow}{D} \psi+\frac{1}{2 m} \int d^{3} x \bar{\psi} \vec{\Sigma} \psi \equiv \vec{\mu}_{L}+\vec{\mu}_{S},
$$

where we have dropped total derivative terms which vanish when taking expectation value in a momentum eigenstate. In the nonrelativistic limit, Eq. (2) reduces to the familiar relation

$$
\vec{\mu}=\frac{1}{2 m}\left(\vec{l}^{(+)}-\vec{l}^{(-)}\right)+\frac{1}{2 m}\left(\vec{\sigma}^{(+)}-\vec{\sigma}^{(-)}\right),
$$

where the superscripts $( \pm)$ denote the contribution from the particle or antiparticle. In Ref. [5], a straightforward relativistic extension of Eq. (3):

$$
\vec{\mu}=\frac{1}{2 m}\left(\vec{L}^{(+)}-\vec{L}^{(-)}\right)+\frac{1}{m}\left(\vec{S}^{(+)}-\vec{S}^{(-)}\right)
$$

where $\vec{S}, \vec{L}$ refer to the relativistic spin and orbital angular momentum, was adopted to calculate the strangeness contribution to $\mu_{N}$. To examine the validity of such straightforward extension, let us recall the relativistic, gauge invariant spin and orbital angular momentum operators of a Dirac field $[6,7]$ :

$$
\vec{S}=\frac{1}{2} \int d^{3} x \psi^{\dagger} \vec{\Sigma} \psi, \vec{L}=\int d^{3} x \vec{r} \times \psi^{\dagger} \frac{1}{2 i} \stackrel{\leftrightarrow}{D} \psi
$$

Comparing Eqs. (2) and (5), we see that the spin and orbital parts of the magnetic moment are not correlated with the spin and orbital angular momentum, but correlated with the tensor charge and the "convection" angular momentum: 


$$
\vec{S}_{\delta} \equiv \frac{1}{2} \int d^{3} x \bar{\psi} \vec{\Sigma} \psi, \vec{L}_{C} \equiv \int d^{3} x \vec{r} \times \bar{\psi} \frac{1}{2 i} \stackrel{\leftrightarrow}{D} \psi
$$

There is an extra $\gamma^{0}$ in $\vec{S}_{\delta}$ and $\vec{L}_{C}$ in comparison to $\vec{S}$ and $\vec{L}$. The effects of this extra $\gamma^{0}$ are at least threefold.

(1) It renders $\vec{S}_{\delta}$ and $\vec{L}_{C}$ charge conjugation odd, thus particle and antiparticle contribute to the magnetic moment with opposite sign.

(2) It implies that if we associate a magnetic moment with $\vec{S}^{( \pm)}$or $\vec{L}^{( \pm)}$, the gyromagnetic ratio would be reversely proportional to the relativistic energy instead of the static mass. In previous studies, we have shown this point explicitly by expanding the operators $\vec{\mu}, \vec{S}$, and $\vec{L}$ in the momentum space [8].

(3) Most importantly, it makes the structure of the quarkantiquark pair creation and annihilation terms (which contribute to the so-called $Z$ diagrams) in $\vec{S}, \vec{L}$ different from that in $\vec{S}_{\delta}, \vec{L}_{C}$, and $\vec{\mu}$ [8]. Numerical studies reveal that both the quark magnetic moment and the quark spin contain significant contributions from the $Z$ diagrams, consequently, Eqs. (3) and (4) are strongly violated [9].

Correlation of the spin magnetic moment $\mu_{S}$ with the tensor charge $\delta q$ makes it possible to determine $\mu_{S}$ experimentally. $\delta q$ can be related to the first moment of the quark transversity distribution $\delta q\left(x, Q^{2}\right)$ [10], which is one of the focuses in the present hadron physics study. Although the chiral-odd $\delta q\left(x, Q^{2}\right)$ decouples from ordinary inclusive deepinelastic scattering (DIS), it may appear together with another chiral-odd object in high-energy hadronic scattering (in particular, Drell-Yan process) or semi-inclusive DIS, and give rise to various double- or single-spin asymmetries. Some single-spin asymmetries have recently been observed in pion production from proton-proton collisions, by the STAR Collaboration at RHIC [11]; and in SIDIS production of pion and/or kaon, by the HERMES Collaboration at DESY using longitudinally polarized target [12], by the SMC Collaboration at CERN using transversely polarized target [13], and by the CLAS Collaboration at JLab using instead unpolarized target and polarized beam [14]. These experiments provided a first glimpse of the transversity distribution. However, interpretation of these measured asymmetries still involves much uncertainties. Some of them might be clarified by upcoming results from RHIC-spin [15], HERMES [16], and COMPASS (CERN) [17]. New ideas of measuring $\delta q\left(x, Q^{2}\right)$ are also continually presented in the literature [18-21].

We emphasize here that the correlation of $\mu_{S}$ with $\delta q$ also brings advantages for lattice QCD calculation. In the present lattice approach, the magnetic moment cannot be computed directly as the expectation value of the operator $\vec{\mu}=\frac{1}{2} \int d^{3} x \vec{r}$ $\times \vec{j}$. This form assumes a center for the nucleon, but on the periodic lattice there is no center for the nucleon due to translational invariance, and the explicit $\vec{r}$ becomes ambiguous at the boundary [22]. In Ref. [23], it was illustrated explicitly that in a periodic system continuum operators which involve powers of the position variable do not project onto good momentum, and so do not isolate the desired matrix element. In practice, the lattice QCD results for magnetic moment are obtained by first computing the magnetic form factor $G_{M}\left(q^{2}\right)$ at finite momentum transfer $q^{2}$, and then extrapolating to $q^{2}=0$. On a finite lattice, the momentum transfer is quantized. In a typical lattice QCD calculation with $\beta=6$ and spatial dimension $16^{3}$, the smallest available nonzero $q^{2}$ is about $0.5 \mathrm{GeV}^{2}$. This makes the extrapolation of lattice QCD results to $q^{2}=0$ a rather awkward task. In fact, the $q^{2} \gtrsim 0.5 \mathrm{GeV}^{2}$ part is usually a flat "tail" of the nucleon form factor, whereas from $q^{2}=0.5 \mathrm{GeV}^{2}$ to $q^{2}=0$ the nucleon form factor might vary dramatically [for example, $G_{M}\left(q^{2}\right)$ increases by a factor of 3]. An extrapolation in such case may naturally introduce large uncertainties. Especially, for quantities where there exists no prior knowledge of their $q^{2}$ dependence, such extrapolation must be strongly model dependent and may even lose its sense.

The tensor charge $\delta q$ does not suffer the above complication, and can be directly computed on the lattice as a forward matrix element. Therefore, computing $\mu_{S}$ via the tensor charge is much better than via the form factors of the spin current in Eq. (1).

In the following, we apply the above discussions to the study of the nucleon strangeness magnetic moment $\mu_{s}$. There are recently great experimental and theoretical efforts to pin down its value [24-26]. This was partially stimulated by the EMC finding that the strange quarks polarize significantly inside the nucleon [1], since such polarization would naturally generate magnetism. Experimentally, $\mu_{s}$ can be determined by measuring the nucleon weak magnetism through parity violating electron-nucleon scattering [24]. The most recent data analysis suggests (with large uncertainty) a positive $\mu_{s}$ [25]. On the contrary, most theoretical calculations typically give a negative $\mu_{s} \sim-0.3$. Normally, one would expect lattice QCD to give the most reliable prediction. However, as we explained above, in the present lattice QCD approach the computation of $\mu_{s}$ is not so accurate. In fact, the results are controversial among various groups [27].

On the other hand, the lattice QCD prediction for the spin part of $\mu_{s}$, or essentially the strangeness tensor charge $\delta s$, can be regarded as relatively reliable. Sadly, in contrast to the numerous lattice QCD and phenomenological studies of $\mu_{s}$, in the literature there appeared just two calculations of $\delta s$, one with lattice QCD [28] and one with chiral quark soliton model [29], which gave $\delta s=-0.046(34)$ and -0.008 , respectively. We therefore feel it necessary to present our own calculation of $\delta s$, with a perturbative meson cloud model which we used in Ref. [9] to compute the strangeness axial charge $\Delta s$. The model Lagrangian

$$
\begin{aligned}
\mathcal{L}= & \bar{\psi}\left[i \not-S(r)-\gamma^{0} V(r)\right] \psi+\frac{1}{2}\left(\partial_{\mu} \phi_{i}\right)^{2}-\frac{1}{2} m_{i}^{2} \phi_{i}^{2} \\
& -\frac{1}{2 F_{\pi}} \bar{\psi}\left[S(r) i \gamma^{5} \lambda^{i} \phi_{i}+i \gamma^{5} \lambda^{i} \phi_{i} S(r)\right] \psi
\end{aligned}
$$

is derived from the nonlinear $\sigma$ model in which meson fields are introduced to restore chiral symmetry [30]; $S(r)=c r+m$ represents the linear scalar confinement potential $\mathrm{cr}$ and the quark mass matrix $m, V(r)=-\alpha / r$ is the Coulomb-type vec- 
TABLE I. The nucleon strangeness tensor charge $\delta s$ calculated with the chiral quark potential model in Ref. [9]. Four sets of widerange parameters are used. Results for the strangeness axial charge $\Delta s$ from Ref. [9] are listed for comparison.

\begin{tabular}{ccccccc}
\hline \hline $\begin{array}{c}\text { Parameter } \\
\text { set }\end{array}$ & $\begin{array}{c}m_{u, d} \\
(\mathrm{MeV})\end{array}$ & $\begin{array}{c}m_{s} \\
(\mathrm{MeV})\end{array}$ & $\alpha$ & $\begin{array}{c}c \\
\left(\mathrm{GeV}^{2}\right)\end{array}$ & & $\delta s$ \\
\hline 1 & 10 & 150 & 0.26 & 0.11 & -0.052 & -0.093 \\
2 & 10 & 150 & 0.26 & 0.16 & -0.070 & -0.102 \\
3 & 300 & 500 & 0.26 & 0.11 & -0.133 & -0.102 \\
4 & 10 & 150 & 0.50 & 0.18 & -0.082 & -0.084 \\
\hline \hline
\end{tabular}

tor potential, $F_{\pi}=93 \mathrm{MeV}$ is the pion decay constant, $\phi_{i}(i$ runs from 1 to 8 ) are the pseudoscalar meson fields, and $\lambda_{i}$ are the Gell-Mann matrices. Here we adopt the same model Lagrangian and the same variety of model parameters as in Ref. [9] and perform an exactly parallel calculation for $\delta s$. Namely, at zeroth order the quark-meson coupling is turned off and the nucleon contains just three valence quarks; then the quark-meson (kaon) coupling is included in lowest order perturbation theory to generate (strange) sea quarks. The calculation results are given in Table I, which are roughly consistent with the range of the lattice QCD prediction in Ref. [28]. For comparison, we also listed values of $\Delta s$ from Ref. [9]. Table I indicates a feature that, being purely sea quark effect, $\delta s$ is not necessarily smaller than $\Delta s$.

A negative $\delta s$ or a negative spin part of $\mu_{s}$ has important implication for the present investigation of $\mu_{s}$. If due to its relative heaviness, the strange quark contributes to $\mu_{N}$ mainly through its polarization, then the total $\mu_{s}$ should also be negative, contrary to the experimental result. On the other hand, if the experimental result of a positive $\mu_{s}$ is confirmed, then the strange quark orbital motion must be important inside the nucleon. We therefore need an updated lattice QCD computation of the quark tensor charge, especially the strangeness sector.

We thank K.F. Liu for illuminating remarks on the lattice QCD calculations. This work was supported in part by the NNSFC (Grant Nos. 90103018, 19975062, 10175033, and 10135030).
[1] For recent reviews of the nucleon spin problem, see R. L. Jaffe, Int. J. Mod. Phys. A 18, 1141 (2003); B. W. Filippone and X. D. Ji, Adv. Nucl. Phys. 26, 1 (2001); M. Anselmino, hep-ph/0302006; H. Y. Cheng, Nucl. Phys. A684, 299 (2001); Chin. J. Phys. (Taipei) 38, 753 (2000). For earlier reviews, see H. Y. Cheng, Int. J. Mod. Phys. A 11, 5109 (1996); U. Stiegler, Phys. Rep. 277, 1 (1996); R. L. Jaffe, Phys. Today 48(9), 24 (1995); M. Anselmino, A. Efremov, and E. Leader, Phys. Rep. 261, 1 (1995).

[2] R. Nag, Prog. Theor. Phys. 91, 409 (1994).

[3] X. Artru, hep-ph/0207309; in Proceedings of RIKEN BNL Research Center Workshop on Future Transverse Measurements, edited by D. Boer and M. G. Perdekamp (BNL, Brookhaven, 2000), Vol. 29.

[4] J. D. Bjorken and S. D. Drell, Relativistic Quantum Mechanics (McGraw-Hill, New York, 1964).

[5] P. Geiger and N. Isgur, Phys. Rev. D 55, 299 (1997).

[6] X. Ji, Phys. Rev. Lett. 78, 610 (1997).

[7] X. S. Chen and F. Wang, Commun. Theor. Phys. 27, 121 (1997).

[8] X. S. Chen, D. Qing, and F. Wang, Chin. Phys. Lett. 16, 403 (1999); D. Qing, X. S. Chen, and F. Wang, Phys. Rev. D 58, 114032 (1998); Phys. Rev. C 57, R31 (1998).

[9] X. B. Chen, X. S. Chen, and F. Wang, Phys. Rev. Lett. 87, 012001 (2001); X. Chen et al., hep-ph/0003158.

[10] For a comprehensive introduction, see V. Barone, A. Drago, and P. G. Ratcliffe, Phys. Rep. 359, 1 (2002); V. Barone and P. G. Ratcliffe, Transverse Spin Physics (World Scientific, Singapore, 2003).

[11] J. Adams et al., STAR Collaboration, hep-ex/0310058.

[12] A. Airapetian et al., HERMES Collaboration, Phys. Lett. B 562, 182 (2003); Phys. Rev. Lett. 84, 4047 (2000); Phys. Rev. D 64, 097101 (2001).
[13] A. Bravar, SMC Collaboration, Nucl. Phys. B (Proc. Suppl.) 79A, 520 (1999).

[14] H. Avakian et al., CLAS Collaboration, hep-ex/0301005.

[15] L. C. Bland, in Proceedings of 15th International Spin Physics Symposium, edited by Y. I. Makdisi, A. U. Luccio, and W. W. MacKay, AIP Conf. Proc. No. 675 (AIP, Melville, NY, 2003), p. 98; G. Bunce, N. Saito, J. Soffer, and W. Vogelsang, Annu. Rev. Nucl. Part. Sci. 50, 525 (2000).

[16] N. C. R. Makins, HERMES Collaboration, Nucl. Phys. A711, 41 (2002).

[17] J. M. Le Goff, COMPASS Collaboration, Nucl. Phys. A711, 56 (2002); M. Lamanna, COMPASS Collaboration, ibid. A711, 50 (2002).

[18] D. Boer, hep-ph/0312320.

[19] J. Soffer, hep-ph/0310195.

[20] E. Di Salvo, Int. J. Mod. Phys. A 18, 5277 (2003).

[21] S. J. Brodsky, D. S. Hwang, and I. Schmidt, Phys. Lett. B 530, 99 (2002).

[22] K. F. Liu (private communications).

[23] W. Wilcox, Phys. Rev. D 66, 017502 (2002).

[24] For reviews, see W. M. Alberico, S. M. Bilenky, and C. Maieron, Phys. Rep. 358, 227 (2002); D. H. Beck and R. D. McKeown, Annu. Rev. Nucl. Part. Sci. 51, 189 (2001); D. H. Beck and B. R. Holstein, Int. J. Mod. Phys. E 10, 1 (2001).

[25] D. T. Spayde et al., SAMPLE Collaboration, nucl-ex/0312016; T. M. Ito et al., Phys. Rev. Lett. (to be published); R. Hasty et al., Science 290, 2117 (2000); D. T. Spayde et al., Phys. Rev. Lett. 84, 1106 (2000); B. Mueller et al., ibid. 78, 3824 (1997).

[26] K. A. Aniol et al., HAPPEX Collaboration, Phys. Lett. B 509, 211 (2001); Phys. Rev. Lett. 82, 1096 (1999).

[27] R. Lewis, W. Wilcox, and R. M. Woloshyn, Phys. Rev. D 67, 013003 (2003); D. B. Leinweber, ibid. 69, 014005 (2004); 
K. F. Liu, J. Phys. G 27, 511 (2001); N. Mathur et al., Kentucky Field Theory Collaboration, Nucl. Phys. B (Proc. Suppl.) 94A, 311 (2001); D. B. Leinweber and A. W. Thomas, Phys. Rev. D 62, 074505 (2000); S. J. Dong, K. F. Liu, and A. G. Williams, ibid. 58, 074504 (1998).
[28] S. Aoki, M. Doui, T. Hatsuda, and Y. Kuramashi, Phys. Rev. D 56, 433 (1997).

[29] H. C. Kim, M. V. Polyakov, and K. Goeke, Phys. Lett. B 387, 577 (1996).

[30] See, e.g., A. W. Thomas, Adv. Nucl. Phys. 13, 1 (1984). 